\title{
Measurements on the Rate of Secondary Recrystallization in High Purity Silver
}

\author{
by F. D. Rosi \\ and B. H. Alexander
}

\begin{abstract}
$T^{1}$ HIS investigation is one of a series primarily designed to measure the rate of growth of secondary grains at various temperatures for a number of high-purity metals ( $\mathrm{Al}, \mathrm{Ag}$ and $\mathrm{Cu}$ ). It is hoped that this will lead to a better understanding of the underlying atomic processes governing this recrystallization reaction.

In this first group of experiments, measurements have been made of the secondary transformation in high purity silver $(99.99+)$ in the form of cold-rolled sheets $0.02 \mathrm{in}$. thick. Annealing these sheets in the temperature range of $433^{\circ}$ to $533^{\circ} \mathrm{C}$ produced a primary recrystallized structure with a grain size of the order of 0.02 $\mathrm{mm}$. On prolonged heating in this temperature range, secondary crystals appeared at various points ranging in size up to approximately $2 \mathrm{~mm}$ diam. Generally speaking, these crystals exhibited a preferential growth in the direction of rolling and showed corrugated boundaries demonstrating visibly that the growth is proceeding preferentially along grain boundaries in accordance with the principles of boundary migration.

The results of the growth velocity measurements showed that the rate of growth, $V$, is invariant with time similar to the growth rate of primary grains in a uniformly deformed test piece. Furthermore, it was established that the temperature dependence of the average velocity of invisible grain growth (reciprocal of "induction for Sylvania Electric Products, Inc., Bayside, L. I.
\end{abstract}

period") as well as the time independent velocity of visible grain growth may be given by the expression ( $\left.\mathrm{V}=\mathrm{V}_{0} \mathrm{e}^{-\mathrm{Q} / R \mathrm{~T}}\right)$.

Thus, the velocity of grain growth in secondary recrystallization corresponds with the growth rate of most processes in solids involving relative movements of atoms.

Activation energies have been calculated describing the temperature dependence of both the visible and invisible growth processes. These values strongly suggest that the underlying atomic process of crystal growth is one of grain boundary self-diffusion. These results further suggest that grain growth is taking place during the so-called induction period by the same processes governing the growth of visible grains, and that this early-stage growth is probably impeded by the small number of contact areas between the growing grain and its surrounding finegrained structure.

Some studies have been made of the orientation relationships between the deformed and recrystallized structures. It was found that the secondary grains exhibited an orientation similar to the deformation texture. This indicates that the nuclei responsible for secondary recrystallization are formed during the deformation process, and that the orientation dependence of the secondary crystals is responsible for their growth.

Experiments are also being conducted to study the effect of grain size of the primary recrystallized structure on the growth velocity of secondary grains.

\section{The Plastic Behavior of Silver Single Crystals at Various Temperatures}

\author{
by F. D. Rosi
}

$T^{\mathrm{s}}$ HE plastic properties of silver single crystals $1(99.99+)$ have been investigated at room temperature $\left(295^{\circ} \mathrm{K}\right)$ and the temperature of dry ice $\left(205^{\circ} \mathrm{K}\right)$ for small amounts of extension ( $\max 2$ pct). Determinations of critical resolved shear stress and coefficient of shear hardening were made, and both properties were found to increase with a decrease in temperature. Furthermore, these properties were consistent with the Schmid law of critical shear stress governing the initiation of the slip process. Values of the elastic modulus at $295^{\circ} \mathrm{K}$ showed a marked dependence on the crystallographic orientation.

At both temperatures the stress-strain diagrams demonstrate a rather sharp advent of plastic flow, and an almost linear rate of strain hardening. In all cases, the hardening rate increases after definite values of shear stress and shear strain which are independent of orienta- tion. An analysis has been made of the dependence of this inflection in the shear stress-shear curve on the temperature of testing.

Short time creep tests ( $\max 3$ minutes) were made by interrupting the loading along different portions of the stress-strain curves. These tests have yielded some interesting data correlating the rate of creep with both the rate of strain hardening and the temperature of testing. In addition, an orientation dependence of these curves has been established.

At present this work is being extended to include investigations at $77^{\circ} \mathrm{K}$ and $373^{\circ} \mathrm{K}$. Similar studies are also being made on single crystals of copper and magnesium.

F. D. Rosi is Senior Engineer for Sylvania Electric Products, Inc., Bayside, L. I. 